\title{
BENEFICIOS DA SALA INTERATIVA NO ESPAÇO DAS PROFISSÕES PARA FORMAÇÃO DO BIOMÉDICO
}

\author{
Karla Lima Rodrigues ${ }^{1,2}$; JoÃo Batista Dutra ${ }^{1}$; Romes Bittencourt \\ Sousa $^{1}$; Francis Patricio Ferreira ${ }^{1}$; Vanessa Canduz Poli ${ }^{1}$; Jeovino \\ Sousa Junior ${ }^{1}$; Neila Teixeira Lima ${ }^{1}$; Roberta Naves Machado ${ }^{1}$; Caio \\ César Araújo ${ }^{1}$; Gustavo Ayres ${ }^{1}$; Dione Lis Souza ${ }^{1}$; Joyce Ventura ${ }^{1}$; \\ Júlia Cristina Cunha ${ }^{1}$; Renata Dias ${ }^{1}$; Vinicius Rodrigues ${ }^{1}$; Gabriela \\ Silvério Bazilio ${ }^{1}$; Renan Nunes Leles ${ }^{1}$; Gustavo Rodrigues Pedrino ${ }^{1}$; \\ Ana Cristina Rebelo ${ }^{1,3}$.
}

\begin{abstract}
Resumo: A Universidade Federal de Goiás realiza anualmente o Espaço das Profissões, evento que promove integração da comunidade acadêmica e a comunidade externa. A sala interativa do curso de biomedicina é um espaço para expor diferentes áreas de atuação do biomédico demonstrando fatos sobre a graduação e a profissão. $\mathrm{O}$ objetivo da ação foi levar a comunidade externa o conhecimento adquirido na graduação, promover interação entre os discentes do curso e o público e permitir que ambos tenham contato com as habilitações do biomédico. Mais 20 habilitações foram representadas por atividades interativas e experimentais. Houve uma resposta positiva do público, que se mostrou bastante interessado e entusiasmado com as apresentações temáticas. Os discentes de Biomedicina se mostraram proativos, motivados e comprometidos com sua futura profissão. Foi possível observar a importância de ações de extensão que permitem aproximação da universidade a comunidade externa e despertar o interesse dos visitantes.
\end{abstract}

Palavras-chave: Espaço das profissões, biomedicina, interação social, Extensão.

\footnotetext{
1 Instituto de Ciências Biológicas, Universidade Federal de Goiás - UFG, Goiânia/GO.

2 karlalrodrigues@outlook.com

3 anacristina.silvarebelo@gmail.com
} 


\begin{abstract}
The Federal University of Goias annually holds the Space of Professions, an event that promotes the integration of the academic community and an external community. The interactive room of the biomedicine course is a space to expose different areas of biomedical performance demonstrating facts about a graduation and a profession. The objective of the action is to take an external community of the acquirer in the graduation, to promote the interaction between the students of the course and the public and to allow the contact with the qualifications of the biomedical one. More 20 qualifications with represented by interactive and experimental activities. There was a positive response from the audience, who is very knowledgeable and enthusiastic about the thematic presentations. The speeches of Biomedicine are proactive, motivated and committed to their future profession. It was possible to observe an importance of extension actions that approach the university of an external community and arouse the interest of the visitors.
\end{abstract}

Key words: Space of the professions, biomedicine, social interaction, Extension.

Resumen: La Universidad Federal de Goiás realiza anualmente el Espacio de las profesiones, evento que promueve la integración de la comunidad académica y una comunidad externa. La sala interactiva del curso de biomedicina es un espacio para exponer diferentes áreas de actuación del biomédico demostrando hechos sobre una graduación y una profesión. El objetivo de la acción es llevar una comunidad externa del adquirente en la graduación, promover la interacción entre los discentes del curso y el público y permitir el contacto con las cualificaciones del biomédico. Más 20 habilitaciones con representadas por actividades interactivas y experimentales. Hubo una respuesta positiva del público, que está muy bien informado y entusiasmado con las presentaciones temáticas. Los discursos de Biomedicina se muestran proactivos, motivados y comprometidos con su futura profesión. Fue posible observar una importancia de acciones de extensión que se aproximen a la universidad de una comunidad externa y despertar del interés de los visitantes.

Palabras clave: Espacio de las profesiones, biomedicina, interacción social, Extensión.

\title{
INTRODUÇÃO
}

Possuir um curso superior nos dias de hoje é sinônimo de ascensão, de garantia de melhores condições de vida e estabilidade financeira (BORGES; CARNIELLI, 2005; SOARES, 2007; NUNES, 2014). Assim, a obtenção de um diploma é tido como um divisor de águas entre o sucesso e o fracasso (OLIVEIRA, 2004; AZEVEDO; FARIA, 2006). Escolher a futura profissão é 
um dos momentos cruciais da vida do estudante, e estar bem preparado na hora de fazer essa escolha pode ajudar o jovem a ter mais sucesso e segurança na sua vida universitária e profissional (ESPAÇO DAS PROFISSÕES UFG, 2010). Ademais entre os diferentes espaços de construção do conhecimento, a Universidade ocupa um lugar privilegiado de convivência e desenvolvimento humano, científico-tecnológico e social (PIVETTA et al., 2010).

Conforme salienta SALDANHA et al, 2008, a escolha de um curso de graduação tornou-se um dilema, pois os jovens têm entrado muito cedo nas universidades. Alguns, por vezes, entram sem saber se tem afinidade ao curso escolhido ou até mesmo sem conhecerem a futura profissão. Estudos já relataram que é necessário experimentar, ou ao menos, buscar o conhecimento de uma profissão, e suas habilitações e os papeis que esta desenvolve na sociedade. Desta maneira essa experiência permitiria uma visão mais realista com o cotidiano de um profissional da área levando ao candidato/aprendiz a saber se terá algum indicio de afinidade com a profissão que se pretende seguir (WHITAKER, 1985; BOMTEMPO, 2005; PINHEIRO 2008; BOMTEMPO, 2012). A falta de conhecimento e afinidade, por vezes, é responsável pela evasão acadêmica (HOTZA; LUCHIARI, 1998). Neste contexto, BOHOSLAVSKY (1981) aponta que a sociedade em que vivemos nos oferece uma visão estereotipada e leviana das profissões, fazendo com que os jovens que acabaram de ingressar em uma universidade sofram um choque de realidade, percebendo que aquela área de atuação não correspondia ao esperado.

Levar o conhecimento para a comunidade é o principal papel da universidade enquanto instituição de ensino. Segundo RODRIGUES et al 2013, "A Extensão Universitária possui papel importante no que se diz respeito às contribuições que pode trazer frente à sociedade." Não obstante, atrair mais pessoas para ingressar nos cursos de graduação deve ser uma atividade secundária da universidade. A Universidade Federal de Goiás (UFG), por meio da Pró-reitora de Graduação, realiza anualmente o "Espaço das Profissões", evento que tem como objetivo a integração da comunidade acadêmica, além de levar a comunidade externa o conhecimento sobre os cursos oferecidos pela instituição - através de palestras e exposições.

Atendendo a 15 mil alunos, o evento foi realizado com sucesso pela primeira vez em setembro de 2009, o qual promoveu, e promove anualmente, o encontro de estudantes do ensino médio com universitários e profissionais da UFG de diversas áreas do conhecimento (ESPAÇO DAS PROFISSÕES UFG, 
2010). Durante o evento, o estudante tem acesso às salas interativas, onde vivencia aspectos das profissões com professores acadêmicos de diversas áreas através de mini palestras, oficinas e apresentações culturais de teatro, dança e música (ESPAÇO DAS PROFISSÕES UFG, 2012). Atualmente mais de $30 \mathrm{mil}$ alunos foram assistidos no espaço das profissões sendo esta uma oportunidade para o aluno do ensino médio de conhecer alguns aspectos da estrutura da Universidade, assim como cursos por ela oferecidos.

Discentes do curso de Biomedicina da UFG, juntamente com sua coordenação trouxeram a Sala Interativa, espaço que foi criado com o intuito de difundir as possíveis áreas de atuação do biomédico, que atualmente abrangem mais de 30 especialidades (CFBM, 2015), trazendo para próximo da comunidade um pouco das atividades diárias realizadas pelos profissionais das diferentes habilitações, desmistificando o senso comum de que o biomédico atua apenas nas análises clínicas. As visitas da sala interativa da biomedicina da UFG buscaram envolver as escolas do Estado Goiás, de rede pública e privada, desde as mais distantes da capital, até aquelas ao redor, de maneira a proporcionar o entendimento do estudante sobre o que é a Universidade, como é o curso, como é o acesso, além de identificar e valorizar talentos, ampliando ainda mais o diálogo com estes jovens e atingi-los em grande escala.

O objetivo da ação foi levar ao público externo parte do conhecimento que é adquirido durante a graduação, promovendo a interação entre os alunos do curso e o público, e por outro lado, permitir que os alunos e a população tenham um contato mais próximo com as habilitações do biomédico. O presente trabalho teve como objetivo verificar, por meio de metodologia qualitativa, a influência da sala interativa na decisão de escolha do curso superior dos visitantes e a permanência dos alunos da graduação ao curso de biomedicina da UFG.

\section{MATERIAIS E MÉTOdos}

Mais de 20 especialidades da biomedicina foram contempladas através de diferentes tipos de exposições, desde apresentações orais até experimentações práticas de procedimentos comuns do cotidiano do biomédico.

Para a exemplificação das habilitações foram utilizados materiais técnicos indispensáveis a atuação do biomédico, tais como microscópios, lâminas histológicas, culturas de microrganismos, espécimes de parasitos e equipamentos utilizados em laboratórios das aulas práticas e de pesquisa. Além disso, 
procedimentos clínicos e experimentais de baixa complexidade foram executados, necessitando de materiais de consumo como reagentes, materiais perfurantes descartáveis e substâncias utilizadas para antissepsia e funcionamento dos experimentos.

Com relação às atividades experimentais foram oferecidas as estações as quais haviam alunos responsáveis por buscar o conhecimento com profissionais da área, afim de conhecer o cotidiano da especialidade. Desta forma os alunos conseguiriam ter uma vivência e conseguiriam passar o conhecimento tanto aos colegas como para o público visitante. As estações ou mesas interativas buscaram interagir diretamente com o público, sendo que muitas das atividades eram necessários o uso de voluntários para os experimentos e explanação dos conteúdos.

A primeira estação era destinada à Pesquisa científica onde o tema dos anos tanto de 2016 como 2017 foi de neurofisiologia e cardiofisiologia, devido a facilidade de obtenção dos equipamentos e realização dos protocolos. Os discentes responsáveis por essa mesa interativa, fizeram visitações ao Centro de Pesquisa em neurociência e Fisiologia Cardiovascular - CPNFC, laboratório do departamento de Ciências Fisiológicas - DCIF do Instituto de Ciências Biológicas - ICB da UFG, com o intuito de conhecer a realidade de um laboratório de pesquisa, além de aprenderem a fundamentação teórica e as técnicas que seriam utilizadas durante o evento. Foram aprendidas as técnicas de estimulação elétrica da musculatura do antebraço, a qual utilizou-se um aparelho com eletrodos bipolares que mimetizam o impulso nervoso responsável pela contração muscular, desta forma pudemos demostrar também o fenômeno de tetânia que é a contração máxima de um musculo. A aferição frequência cardíaca foi a outra técnica e para isso utilizamos um transdutor de pressão arterial pulsátil, que capta sinais mecânicos e os envia para um conversor analógico digital (Powerlab System, ADInstruments, Colorado Springs, CO, USA) que posteriormente são calculados pelo software Labchat (Chart v7.3.7, ADInstruments, Colorado Springs, CO, USA) para obtenção dos valores dos batimentos cardíacos.

Na habilitação de perícia criminal, foi realizada a coleta e análise interativa das impressões digitais dos estudantes visitantes da sala, o intuito foi proporcionar uma experiência prática com dactiloscopia, que é uma das áreas contempladas na ciência forense. Foi feita a análise da morfologia das cristas e sulcos papilares presentes na derme da polpa dos dedos quanto a sua forma 
e direcionamento, sendo nomeados de acordo com a Icnofalangometria em arco, presilha interna, presilha externa e verticilo. Os discentes procuraram se informar com profissionais da área, e ainda buscar o máximo de informações a respeito da especialidade na internet e livros, para que no dia do evento pudessem explicar devidamente ao público de como o perito biomédico pode auxiliar na resolução de crimes.

Também foi feita uma estação sobre as análises clinicas, os alunos responsáveis efetuaram uma visita ao Laboratório de Análises Clinicas e Educação em Saúde - LACES da UFG com supervisão do Prof. Dr. Renan Nunes Leles e a diretora do próprio laboratório a biomédica Gabriela Silvério Bazílio, com a finalidade de aprender os protocolos de biossegurança quando se lida com amostras biológicas e um grande público, além de aprenderem a base teórica e prática da técnica. Colocamos como técnica a tipagem sanguínea, onde fizemos a determinação do tipo sanguíneo do sistema $\mathrm{ABO}$ e do fator Rh de vários visitantes que se voluntariavam. Para isso utilizamos kits de anticorpos que quando entram em contato com a amostra sanguínea aglutinam e a partir disso consegue-se determinar o tipo sanguíneo do indivíduo.

Tendo em vista que o maior campo dentro da biomedicina é o das análises clínicas, e que a bioquímica é a responsável pelo maior movimento dentro de um laboratório clínico, foi criada a estação de bioquímica disponibilizado aos alunos a oportunidade de conhecer um teste bioquímico simples que exemplifica grande parte dos exames que podem ser realizados por um biomédico, foi escolhido o teste de Biureto. O Biureto é um reagente de coloração azul que contém em sua composição Sulfato de Cobre $\left(\mathrm{CuSO}_{4}\right)$, essa molécula se dissocia na presença de água liberando íons $\mathrm{Cu}^{2+}$ que, por sua vez se ligam ao nitrogênio presente nas ligações peptídicas e levam ao desenvolvimento de coloração violeta. Sendo assim uma ótima ferramenta para pesquisa de proteínas em soluções. As soluções escolhidas para a atividade interativa iam desde água pura até soluções de ovalbumina, cistina e glicina.

Durante o espaço das profissões criamos uma estação de radiologia para disseminação da possibilidade da atuação do biomédico como Imagenologista. Recentemente tem-se observado um crescente potencial de inserção de biomédicos na área de Imagenologia, tanto que o Curso de Biomedicina da UFG contempla esse assunto durante o primeiro período através da Anatomia Humana 1 que serve como base para Anatomia Humana 2 que se dedica, mais 
especificamente, ao ensino dos exames de imagem utilizados na clínica médica como Raios X, Tomografia Computadorizada e Ressonância Magnética.

Na estação de Genética, os alunos visitaram laboratórios do departamento de genética - DGEN que se encontra no Instituto de Ciências Biológicas da UFG, onde tiveram contato com profissionais da área de genética e aprenderam a técnica que é utilizada em aulas práticas do curso como a extração de material genético de frutas. O objetivo dessa essa atividade foi entender os conceitos de genética básica e demonstrar como podemos identificar e extrair o DNA do morango/banana como um bom modelo para esse tipo de estudo e atividade prática. Utilizou-se microscópios para visualização de lâminas de divisões celulares as quais foram demostradas também a cariotipagem de células. O público pode obter também como é feito o diagnóstico de alterações cromossômicas como a trissomia do cromossomo vinte e um (Síndrome de Down).

A microbiologia, uma das habilitações o biomédico, se dedica ao estudo dos microrganismos patogênicos, responsáveis pelas doenças infecciosas, englobando a bacteriologia, virologia e micologia, especialidade muito estudada durante a graduação, também foi exposta na sala interativa, onde ocorreu a exposição de cultura de bactérias e fungos, além de que os alunos visitaram vários laboratórios do Instituto de Patologia e Saúde Pública - IPTSP da UFG para aprenderem mais de perto a rotina de um microbiologista e assim explanarem para os visitantes de como esses profissionais biomédicos trabalham e como esses patógenos atuam em diversas doenças humanas.

A especialidade de parasitologia, também foi representada na sala interativa e tivemos a exposição de espécimes dos principais parasitos humanos, assim, procuramos exemplificar para os visitantes que essa ciência estuda os parasitas, os seus hospedeiros e as relações entre eles. O IPTSP que também abriga essa especialidade e deu essa oportunidade para que os alunos da biomedicina pudessem visitar os laboratórios de parasitologia e vivenciassem a rotina e entendessem um pouco mais de como os estudos nessa área não necessários para a saúde pública e dessa forma tiveram subsídio para explicações no dia do Espaço das Profissões.

Um dos maiores símbolos da Biomedicina é a dedicação ao estudo e às análises do líquido sanguíneo e, para que os estudantes pudessem ter contato com a ciência da hematologia fizemos uma estação com essa habilitação. Foi feita avaliação microscópica das células através do esfregaço sanguíneo, técnica básica dentro do hemograma. As lâminas foram preparadas anteriormente, 
e colocadas sob microscópio óptico onde os estudantes podiam observar as estruturas presentes. Foi possível detectar a presença de várias células do sangue: eritrócitos, plaquetas e grande parte dos leucócitos (Neutrófilos, eosinófilos, linfócitos e monócitos).

A histologia é o estudo dos tecidos, sua constituição celular e extracelular. Procuramos expor essa área de estudo do biomédico, pois é um dos pilares da graduação. Por meio da microscopia é possível analisar sua composição e também suas particularidades permitindo diferenciar cada tipo de tecido do corpo. Existem técnicas especiais nas quais um órgão ou tecido macroscópico retirado para biópsia é seccionado, preparado, corado e preservado em forma de lâmina para que seu estudo posterior possa ser feito em microscópio. Tivemos participação do Departamento de Histologia - DHISTO do Instituto de Ciências Biológicas - ICB da UFG que nos forneceram maquetes, microscópios e lâminas com cortes histológicos que são utilizados durante as aulas da graduação.

Por fim, tivemos a habilitação em estética, em que o biomédico atua nos tratamentos estéticos faciais, corporais e capilares. Podendo trabalhar com procedimentos invasivos ou não invasivos, afim de melhorar seu bem-estar de pacientes e consequentemente sua saúde. Os alunos responsáveis por essa habilitação tiveram a experiência de presenciar uma aula teórica/pratica de uma pós-graduação, onde puderam aprender o método de eletroestimulação facial que foi utilizado durante os dias do evento e utilizou os visitantes como voluntários.

A partir dos relatórios anuais de egresso dos discentes do curso de biomedicina da UFG, procuramos fazer um perfil de procura no mercado de trabalho dos alunos recém-formados. Além de informar a população de que o biomédico não atua apenas na área de análises clinicas, que são dezenas de opções disponíveis para os futuros alunos explorarem. Por outro lado, os atuais alunos do curso têm a ciência das suas possibilidades após formados e de como podem desfrutar mais das oportunidades que o mercado lhes oferece.

O evento foi financiado com recursos próprios da coordenação de graduação do curso de Biomedicina, e não contou com a participação financeira de outras instituições.

\section{RESUltados E Discuss Ão}

A coordenação de curso elaborou um relatório da evasão dos discentes, o qual demonstrou o perfil do egresso e sua inserção ao mercado de trabalho 
dos alunos formados em biomedicina na UFG entre os anos de 2013 até 2017. Os resultados mostraram que $18 \%$ está inserido no campo de análises clínicas, $42 \%$ em pesquisa, e 5\% atuando na área de biomedicina estética. Aparecem em menor frequência, as habilitações: acupuntura, perito-auxiliar autópsia, representante de empresa de produtos laboratoriais, docência, saúde pública e reprodução assistida (Tabela 1).

Sobre os resultados dos alunos inseridos no mercado de trabalho, obtivemos que $18 \%$ dos alunos que se formaram entre os anos de 2013 a 2017 estão inseridos no campo de análises clínicas. A habilitação em análises clínicas permite ao profissional biomédico realizar exames, assumir a responsabilidade técnica e firmar os respectivos laudos, executar o processamento de sangue, suas sorologias e exames pré-transfusionais, assumir chefias técnicas, assessorias e direção destas atividades. As principais áreas de análise são a hematologia, a microbiologia, a bioquímica, a parasitologia e a imunologia. Esse ramo tem crescido muito, principalmente nos últimos anos, em tecnologia e inovação, resultando numa demanda cada vez maior de profissionais mais especializados e capacitados (CFBM, 2009). O curso atualmente conta com vários locais para estágio obrigatório no município de Goiânia, que vão desde centros de assistência integral a saúde (CAIS), hospitais como Hospital das Clínicas da UFG, Hospital de Urgência de Goiânia (HUGO), Hospital Maternidade Dona Iris, Centro de Reabilitação e Readaptação Dr. Henrique Santilo (CRER), Hospital Araújo Jorge - Referência em tratamentos de câncer e outros locais como bancos de sangue: Hemolabor e Hemocentro e laboratórios de análises clinicas privados e da própria universidade (Laboratório Margarida Dobller Komma - IPTSP, Laboratório Rômulo Rocha - FF e Laboratório de Análises Clinicas e Ensino em Saúde - LACES.

O campo da pesquisa é uma das áreas clássica e tradicional que perdura por anos, cerca de $42 \%$ dos alunos que se formam seguem por esse caminho. Por vezes dão continuidade a atividades que já faziam durante a graduação no programa de iniciação cientifica, que tem como objetivo despertar vocação científica e incentivar novos talentos entre estudantes de graduação (CNPq, 2006). Além de ser uma oportunidade aos alunos durante o curso poderem vivenciar a realidade e de certa forma trabalhar em uma das 37 áreas de atuação do biomédico (CFBM, 2015). Nas últimas quatro décadas a pesquisa em biomedicina sofreu transformações em sua base de conhecimento tecnocientífico (GAUDILLIÈRE, 2002; CLARKE et al., 2003; KEATING; 
CAMBROSIO, 2003; GUIMARÃES, 2004; BURRI; DUMIT, 2007; LOCK \& GORDON, 2008). Transformações, essas, caracterizadas pela emergência da proteômica, da genômica, da bioinformática e da nanotecnologia e sua associação com a fisiologia, virologia, a bacteriologia e a parasitologia. Essa associação influenciou abordagens científicas e o estudo de doenças associadas multidisciplinarmente (MOREL et al., 2007). Contribuindo assim para o desenvolvimento de novos produtos e processos de produção industrial, além de novas metodologias para a prevenção, o tratamento e o diagnóstico de doenças (BUSS et al., 2005; BUSS et al., 2008).

A biomedicina estética nos últimos anos tem ganhado muito destaque, 5\% dos recém-formados em biomedicina tem escolhido essa habilitação como profissão, em alguns casos trabalham em clinicas ou na docência em pós-graduações de estética. Os procedimentos estéticos "não invasivos e invasivos não-cirúrgicos" são as mais novas habilitações do biomédico. Quem atua neste ramo da saúde, com qualificação em Biomedicina, exerce a atividade como "biomédico esteta". Tais procedimentos estéticos são utilizados para tratamento do processo de envelhecimento cutâneo e disfunções estéticas corporais e faciais. Essas terapias promovem benefícios incomparáveis aos pacientes, incluindo melhor auto-estima, bem-estar e, consequentemente, melhor qualidade de vida. Os recursos aplicados pelos profissionais de biomedicina habilitados compreendem a eletroterapia e eletroestimulação, ultrassonografia em estética, peeling mecânico e peeling químico, carboxiterapia, intradermoterapia, dermaroller, laserterapia, fototerapia, fotorejuvenescimento e preenchimentos semipermanentes. A metodologia desenvolvida baseia-se na revisão bibliográfica e o resultado esperado é disseminar estudos com comprovação científica na comunidade de estudantes e profissionais da saúde (SOUSA, 2012; BATISTA, 2014; FONSECA et al, 2015). 
TABELA1. Inserção no Mercado de Trabalho e Perfil do Egresso

\begin{tabular}{|c|c|c|c|c|c|c|c|}
\hline ATIVIDADE & 2013 & 2014 & 2015 & 2016 & 2017 & TOTAL & Dist. $\%$ \\
\hline $\begin{array}{l}\text { DESISTÊNCIA NO ANO DE } \\
\text { INGRESSO }\end{array}$ & 5 & 6 & 6 & 10 & 10 & 37 & $19 \%$ \\
\hline ANÁLISES CLÍNICAS & 6 & 7 & 9 & 5 & 9 & 36 & $18 \%$ \\
\hline ACUNPUNTURA & 1 & 0 & 0 & 0 & 0 & 1 & $1 \%$ \\
\hline PERITO-AUXILIAR AUTÓPSIA & 3 & 0 & 0 & 0 & 0 & 3 & $2 \%$ \\
\hline PESQUISA & 16 & 18 & 14 & 15 & 19 & 82 & $42 \%$ \\
\hline $\begin{array}{l}\text { CURSANDO OUTRA } \\
\text { GRADUAÇÃO }\end{array}$ & 2 & 3 & 2 & 2 & 2 & 11 & $6 \%$ \\
\hline $\begin{array}{c}\text { EMPRESA DE PRODUTOS } \\
\text { LABORATORIAIS }\end{array}$ & 1 & 0 & 0 & 0 & 0 & 1 & $1 \%$ \\
\hline TRABALHA EM OUTRA ÁREA & 1 & 0 & 0 & 0 & 2 & 3 & $2 \%$ \\
\hline BIOMEDICINA ESTÉTICA & 0 & 3 & 3 & 1 & 3 & 10 & $5 \%$ \\
\hline CONCURSO PÚBLICO & 0 & 0 & 1 & 0 & 0 & 1 & $1 \%$ \\
\hline RESIDENCIA-ESPECIALIZAÇAO & 0 & 0 & 2 & 0 & 4 & 6 & $3 \%$ \\
\hline DocÊNCIA & 0 & 0 & 0 & 1 & 1 & 2 & $1 \%$ \\
\hline SAÚdE PÚBLICA & 0 & 0 & 0 & 1 & 0 & 1 & $1 \%$ \\
\hline REPRODUÇÃO ASSISTIDA & 0 & 0 & 0 & 0 & 1 & 1 & $1 \%$ \\
\hline TOTAL & 35 & 37 & 37 & 35 & 51 & 195 & $100 \%$ \\
\hline CONSECUIU CONTATO & 35 & 37 & 37 & 35 & 51 & 195 & $64 \%$ \\
\hline $\begin{array}{c}\text { NÃO HOUVE CONTATO OU } \\
\text { NÃO FORMOU }\end{array}$ & 25 & 23 & 23 & 25 & 13 & 109 & $36 \%$ \\
\hline TOTAL & 60 & 60 & 60 & 60 & 64 & 304 & $100 \%$ \\
\hline
\end{tabular}

DIST=DISTRIBUIÇÃO; \%=PERCENTUAL

A partir dos relatórios anuais de egresso dos discentes do curso de biomedicina da UFG, procuramos fazer um perfil de inserção no mercado de trabalho dos alunos recém-formados. A partir disso, informar a população de que o biomédico não atua apenas na área de análises clinicas, que são dezenas de opções disponíveis que os futuros alunos podem explorar. Afim de atingir os atuais alunos do curso para terem ciência das suas possibilidades após formados e de como podem desfrutar mais das oportunidades que o mercado lhes oferece. Além de terem um contato com os profissionais das áreas de atuação e terem um contato mais próximo com a área de ensino e docência.

Em 2016 procuramos fazer um remodelamento e reinventar a sala interativa em relação aos anos anteriores, com o intuito de mostrar a sociedade qual é 
a realidade de uma graduação em biomedicina trazendo demonstrações práticas e explanações teóricas e ainda possibilitar uma aplicação de como estas atividades influenciam nas habilitações do biomédico. Ademais, tentamos envolver os discentes a terem um contato mais próximo com essas habilitações e alguns profissionais que já atuam nestas áreas, pois acontece durante o curso de forma lenta e gradual, período após período, durante os anos da graduação.

Assim, durante o evento foi possível perceber uma resposta positiva do público a este modelo, pois se mostraram bastante interessados e entusiasmados com as apresentações temáticas e os alunos do curso de Biomedicina envolvidos na ação se mostraram proativos, motivados e comprometidos com sua futura profissão. Além disso, o número de visitantes em 2016 e 2017 superou os anos anteriores, com aproximadamente 1700 e 2600 visitantes, respectivamente. Uma média superior a 2.000 visitantes por ano, sendo que a média diária chegou a aproximadamente a 1.000 pessoas.

Tem sido muito discutido no meio acadêmico, tentativas de estabelecer parcerias e discutir formas de aproximar os alunos do ensino médio com a Universidade. Estudos mostram a importância de aproximar as pesquisas científicas das universidades e o ensino básico (Lüdke \& Cruz, 2005); além da importância de conhecer os percursos formativos e trilhas percorridas para atravessar o caminho incerto entre a escola pública e a universidade (Sampaio, 2011). Neste contexto, $90 \%$ das visitas da sala interativa, geralmente, são de alunos que frequentam o ensino médio tanto de escolas públicas como colégios privados. Os quais em alguns meses estarão pleiteando uma vaga para ensino superior. A expectativa desses indivíduos no momento de escolha de um curso superior é notável, visto que eles estão prestes a tomar uma decisão que irá interferir em toda sua vida. A escolha profissional geralmente se dá nesta fase da adolescência, período em que esses jovens não estão preparados para uma tomada de decisão deste nível como visto por Miranda, 2001. A partir desta ação, foi possível observarmos o quanto é importante ações de extensão que possibilitam uma relação mais próxima entre a universidade e a comunidade externa, de tal maneira fornecendo informações sobre os cursos da UFG e despertando o interesse destes jovens aos cursos superiores.

O momento de escolha é cercado de variáveis e pressões devido a grande importância que é atribuída a este momento que pode ser considerado o início da transposição para a vida adulta e consequentemente para um futuro bem ou malsucedido. O grande volume de informações, as incertezas 
quanto ao mercado de trabalho e a concorrência para conquistar uma vaga na faculdade são fatores que afetam a procura de um emprego e aumentam a pressão sobre essas escolhas (MYBURGH, 2005; PINHEIRO, 2008; KNOP, 2008). Desta forma, procuramos envolver os alunos da graduação com a extensão universitária e incentiva-los a permanecerem no curso e que as opções no mercado de trabalho são amplas. Além disso, estimular estes discentes à docência e trabalhos que envolvam o ensino.

A coordenação do curso de Biomedicina, principal incentivadora do projeto, considerou o evento como um sucesso, tanto para a comunidade externa quanto para a comunidade acadêmica, uma vez que o projeto despertou interesse nos visitantes e motivou os acadêmicos. Além disso, alguns dissentes puderam ter um primeiro contato com o público externo, o que é de grande relevância, haja visto a natureza de formação do curso.

\section{CONSIDERAÇÕES FINAIS}

Desta maneira, foi possível observar o quanto é importante ações de extensão que levam a universidade um pouco mais próxima da comunidade externa, de tal maneira fornecer informações sobre os cursos da UFG e despertar o interesse dos visitantes ao curso. Além de envolver os alunos da graduação com a extensão universitária e incentiva-los à docência e trabalhos que envolvam o ensino. Ademais, manter os discentes estimulados e entusiasmados com o curso que atualmente cursam em tentativa da redução de evasão do curso.

Em conjunto, através do resultado obtido com o modelo de sala interativa no ano de 2016 e 2017, o que se espera é a repetição dos resultados ou até mesmo a melhora dos mesmos, ampliação do alcance na comunidade externa à Universidade e aumento na adesão dos acadêmicos do curso de Biomedicina ao projeto e ao curso.

\section{REFERÊNCIAS}

AZEVEDO, A. S.; FARIA, L. Motivação, sucesso e transição para o ensino superior. Psicologia, v.20, n.2, p.69-93, 2006.

BATISTA, Juliana Alves. Pós-graduação em Estética. Revista do Biomédico. São Paulo. $n$ 113. Nov.- dez., 2014.

BOHOSLAVSKY, R. Orientação profissional: a estratégia clínica. São Paulo, 1977. 
BOMTEMPO, M. S.; SILVA, D.; FREIRE O. Motivos da escolha do curso de administração de empresas por meio da modelagem de equações estruturais. V $13 \mathrm{n}^{\circ} 3 \mathrm{Jul} /$ Set. Belo Horizonte, 2012.

BOMTEMPO, M.S. Análise dos fatores de influência na escolha pelo curso de graduação em Administração: um estudo sobre as relações de causalidade, através da modelagem de equações estruturais. 2005. 142 p. Dissertação Mestrado em Administração de Empresas - Fundação Escola de Comércio Álvares Penteado - FECAP, São Paulo, 2005.

BORGES, J. L.; Carnielli, B. L. Educação e estratificação social no acesso à universidade pública. Cadernos de Pesquisa, 35(124), 113-139, 2005.

BURRI, R. V.; DUMIT, J. (Eds.). Biomedicine as culture: instrumental practices, technoscientific knowledge, and new modes of life. New York: Routledge, 2007. p.251, 2007. BUSS, P.M.; CARVALHEIRO, J.R.; CASAS, C.P.R. (Org.). Medicamentos no Brasil: inovação e acesso. Rio de Janeiro: Editora Fiocruz, p.440, 2008.

CFBM - Conselho Federal de Biomedicina. Biomedicina: Um painel sobre o profissional e a profissão. Livro. 76 f. 2009.

CFBM - Conselho Federal de Biomedicina. Habilitações. 2015. Disponível em <http:// cfbm.gov.br/habilitacao/>. Acesso em 14 de fevereiro de 2018.

CLARKE, A. E., Shim, J. K., MAMO, L., FOSKET, J. R., Jennifer, R., \& ClARKE, A. E. Biomedicalization: technoscientific transformations of health, illness, and u.s. biomedicine. American Sociological Review, v.68, n.2, p.161-194, 2003.

CNPq - Conselho Nacional de Desenvolvimento Científico e Tecnológico, Objetivos do Programa PIBIC, 2006. Disponível em: $\langle$ http://cnpq.br/pibic $>$. Acesso em 26 de janeiro de 2018.

ESPAÇO DAS PROFISSÕES UFG. Disponível em <http://vestibular.brasilescola.com/ noticias/ufg-recebe-inscricoes-para-espaco-das-profissoes-2012/318894.html >. Acesso em 14 de fevereiro de 2018.

ESPAÇO DAS PROFISSÕES UFG. Disponível em <http://www.vestibular.ufg.br/2013/ $\mathrm{EP} />$. Acesso em 25/02/2018.

FONSECA, L. P.; Couto, R. M.; COSTA, E. G. D.; COSTA, E. G. D. Legislação estética. Anais do XVII Encontro de Iniciação Científica e II Mostra de Extensão da Unin Cor. p 103. Três corações - MG, 2015.

GAUDILLIÈRE, J.-P. Inventer la biomédicine: la france, lamérique et la production des savoirs du vivants (1944-1965). Paris: La Découverte, p.392, 2002.

GUIMARÃES, Jorge A. A pesquisa médica e biomédica no Brasil. Comparações com o desempenho científico brasileiro e mundial Ciência \& Saúde Coletiva, 9 (2):303-327, 2004.

HOTZA, M. A. S. A re-escolha profissional dos vestibulandos da UFSC de 1997. Revista da ABOP, 2(1), 97-110, 1998. 
KEATING, P.; CAMBROSIO, A. Biomedical platforms: realigning the normal and the pathological in late-twentieth-century medicine. Cambridge, MA: The MIT Press, p.560, 2003.

KNOP, M. N. H. A escolha de curso superior dos vestibulandos da Universidade Federal do Rio Grande do Sul: um estudo quantitativo com utilização de Análise de Correspondência Múltipla. 2008, 130 p. Dissertação de Mestrado em Sociologia - UFRS, Porto Alegre, 2008.

LOCK, M.; GORDON, D. (Eds.). Biomedicine examined. New York: Springer, p.572, 2008.

LÜDKE, M.; CRUZ, G. B. Aproximando universidade e escola de educação básica pela pesquisa. Cadernos de pesquisa, 35 (125), 81-109, 2005.

MOREL, C. M., Carvalheiro, J. R., Romero, C. N., Costa, E. A., \& BUSS, P. M. The road to recovery. Nature, v.449. p.180-182, 2007.

MYBURGH, J. E. An enpirical analysis of career choce factors that influence first-year Accouting students at the University of Pretoria: a cros-racial study. Meditari Accountancy Reserarch, (S.I.), v. 13, n. 2, p 35-48, 2005.

NUNES, C.A. Fatores determinantes na escolha pelo curso de ciências contábeis em IES particulares da cidade de São Paulo. 89 p. Dissertação de Mestrado em Ciências Contábeis - Fundação e Escola de Comércio Álvares Penteado - FECAP, São Paulo, 2014.

OLIVEIRA, L. R. Reflexões sobre os desafios da educação superior. Análise. Revista da Faculdade de Ciências Econômicas, Contábeis e de Administração de Empresas Padre Anchieta. Jundiaí-SP: Sociedade Padre Anchieta de Ensino. 23 cm., 25, 2004.

PINHEIRO, R. G. Fatores de escolha pelo curso de Ciências Contábeis: uma pesquisa com os graduandos na capital e Grande São Paulo. 111 p. Dissertação de mestrado em Ciências Contábeis - Fundação e Escola de Comércio Álvares Penteado - FECAP, São Paulo, 2008.

PIVETTA, H. M. F., STEIN Backes, D., CARPES, A., HOLANDA Tavares Battistel, A. L., \& MARCHIORI, M. Ensino, pesquisa e extensão universitária: em busca de uma integração efetiva. Linhas Críticas, Brasília, DF, v. 16, n. 31, p. 377-390, jul./dez. 2010.

RODRIGUES, A. L. L., do Amaral Costa, C. L. N., PRATA, M. S., BATALHA, T. B. S., \& NETO, I. D. F. P. Contribuições da extensão universitária na sociedade. Caderno de Graduação-Ciências Humanas e Sociais-UNIT, 1(2), 141-148, 2013.

SALDANHA, L. A.; SILVA, J. R. \& CASTRO, S. M. R. (2007). Sonhos e crises: Marcas da jornada universitária. Disponível na World Wide Web pela URL $<$ http://www.jussara. ueg.br/pos_docencia_universitaria>. Acesso em 19 de janeiro 2018.

SAMPAIO, SMR., org. Entre a escola pública e a universidade: longa travessia para jovens de origem popular. In: Observatório da vida estudantil: primeiros estudos. Salvador: EDUFBA, pp. 27-51. ISBN 978-85-232-1211-7, 2011. 
SOARES, S. A. M. A escolha do curso de serviço social: implicações subjetivas. 102 p. Dissertação de Mestrado em Serviço Social - Pontifícia Universidade Católica de São Paulo - PUC-SP, São Paulo, 2007.

SOUSA, Poliana Mendonça de. A atuação do biomédico em procedimentos estéticos não invasivos e invasivos não cirúrgicos. 2012. 38 f. Monografia (Graduação) - Universidade Católica de Brasília, Brasília, 2012.

WHITAKER, D. C. A. A escolha da carreira. São Paulo, SP: Moderna, 1985. 